\title{
The Antineoplastic Activity of Photothermal Ablative Therapy with Targeted Gold Nanorods in an Orthotopic Urinary Bladder Cancer Model
}

\author{
Xiaoping Yang ${ }^{\mathrm{a}, \mathrm{e}}$, Lih-Jen Su ${ }^{\mathrm{a}}$, Francisco G. La Rosa ${ }^{\mathrm{b}, \mathrm{e}}$, Elizabeth Erin Smith ${ }^{\mathrm{b}, \mathrm{e}}$, \\ Isabel R. Schlaepfer ${ }^{\mathrm{a}}$, Suehyun K. Cho ${ }^{\mathrm{d}}$, Brian Kavanagh ${ }^{\mathrm{c}}$, Wounjhang Park $^{\mathrm{d}}$ \\ and Thomas W. Flaiga,e,* \\ ${ }^{a}$ Department of Medicine, Division of Medical Oncology, University of Colorado School of Medicine, Aurora, \\ CO, USA \\ ${ }^{\mathrm{b}}$ Department of Pathology, University of Colorado School of Medicine, Aurora, CO, USA \\ ${ }^{\mathrm{c}}$ Department of Radiation Oncology, University of Colorado School of Medicine, Aurora, CO, USA \\ ${ }^{\mathrm{d}}$ Department of Electrical, Computer and Energy Engineering, University of Colorado, Boulder, CO, USA \\ ${ }^{\mathrm{e}}$ University of Colorado Cancer Center, Aurora, CO, USA
}

\begin{abstract}
. irradiated with NIR light in an orthotopic urinary bladder cancer model. pre-treatment levels $(p=0.045)$. tolerated in a murine in vivo model of urinary bladder cancer. intravesical

${ }^{*}$ Correspondence to: Thomas W. Flaig, Department of Medicine, Division of Medical Oncology, University of Colorado School of Medicine, Aurora, CO 80045, USA. E-mail: Thomas.Flaig@ucdenver.edu.
\end{abstract}

Background: Gold nanoparticles treated with near infrared (NIR) light can be heated preferentially, allowing for thermal ablation of targeted cells. The use of novel intravesical nanoparticle-directed therapy in conjunction with laser irradiation via a fiber optic cystoscope, represents a potential ablative treatment approach in patients with superficial bladder cancer.

Objective: To examine the thermal ablative effect of epidermal growth factor receptor (EGFR)-directed gold nanorods

Methods: Gold nanorods linked to an anti-EGFR antibody (Conjugated gold NanoRods - CNR) were instilled into the bladder cavity of an orthotopic murine xenograft model with T24 bladder cancer cells expressing luciferase. NIR light was externally administered via an $808 \mathrm{~nm}$ diode laser. This treatment was repeated weekly for 4 weeks. The anti-cancer effect was monitored by an in vivo imaging system in a non-invasive manner, which was the primary outcome of our study.

Results: The optimal approach for an individual treatment was $2.1 \mathrm{~W} / \mathrm{cm}^{2}$ laser power for 30 seconds. Using this in vivo model, NIR light combined with CNR demonstrated a statistically significant reduction in tumor-associated bioluminescent activity $(n=16)$ compared to mice treated with laser alone $(n=14)$ at the end of the study $(p=0.035)$. Furthermore, the CNR+NIR light treatment significantly abrogated bioluminescence signals over a 6-week observation period, compared to

Conclusions: Photothermal tumor ablation with EGFR-directed gold nanorods and NIR light proved effective and well

Keywords: Urinary bladder neoplasms, metal nanoparticles, laser therapy, near-infrared (NIR) light, administration,

\section{INTRODUCTION}

Urinary bladder cancer is common, with more than 75,000 cases expected and over 16,000 deaths in the United States in 2016 [1]. Most cases (70\%) present 
as superficial, non-muscle invasive disease. One unique feature in the treatment of superficial urinary bladder cancer is the ability to deliver medical therapy directly to bladder tumors via a urinary catheter. This approach may also limit systemic drug exposure minimizing the toxic impact of therapy. Intravesical bacillus Calmette-Guérin (BCG), administered into the bladder by routine urinary catheterization, has been a standard approach to non-muscle invasive bladder cancer for decades. Despite BCG treatment, recurrent and progressive disease leading to cystectomy or metastatic disease is common [2].

Recently, the use of gold nanorods (nanotechnology) has become an area of increased research investigation, including therapeutic applications $[3,4]$. However, with systemic delivery of nanoparticles, concerns about hepatic and renal clearance have been raised $[5,6]$. In seeking a differentiallyexpressed cell surface tumor marker in bladder cancer, the epidermal growth factor receptor (EGFR), which is frequently over-expressed on luminal surface of bladder cancer cells [7-9], as opposed to the normal urothelium, appears to be a good therapeutic targeting candidate. Based on the current clinical use of fiber-optic cystoscopes [10], the use of novel intravesical nanoparticle-directed therapy in conjunction with photothermal laser irradiation via a fiberoptic cystoscope, represents a clinically feasible treatment approach in patients with superficial bladder cancer. This would not replace the use of current cystoscopic debulking procedures, but rather augment these by using this approach after such procedures to treat any residual microscopic disease remaining. In a previous study [11], we described the preparation of Conjugated gold NanoRods (CNR) with addition of an anti-EGFR antibody and its application in vitro using the HTB9 bladder cancer cell model. In this study, we examine the safety and efficacy of EGFRdirected nanoparticle thermal ablation therapy in vivo using an orthotopic xenograft mouse model. T24 human cells were selected based on their expression of EGFR [12] and suitability for orthotopic implantation, which was critical to these intravesical experiments. While the eventual clinical application would provide laser delivery via a cystoscopic means, we use external delivery in this model, as NIR has adequate tissue penetration to reach all aspects of the murine bladder (reviewed herein [13]). We have previously developed this orthotopic model of bladder cancer with T24 human bladder cancer cells, using a bioluminescent response endpoint, for which a response was well correlated to pathologic response [12].

\section{MATERIALS AND METHODS}

\section{Materials, laser and cells}

Gold nanorods and CNR were prepared by the method described before [11]. An $808 \mathrm{~nm}$ diode laser (DILAS MINI 808-50/400) was purchased from Dilas Diode Laser Inc. (Tucson, AZ). T24 cells were obtained from ATCC (Manassas, VA). T24 cells with luciferase labeling were prepared following the previously reported method [12]. Cell line authentication was done by UCCC DNA Sequence $\&$ Analyses Core, using ABI Identifiler kit. The loci tested are the following : D3S1358, vWA, FGA, Amelogenin, D8S1179, D21S11, D18S51, D5S818, D13S137, D7S820, CSF1PO, D16S539, THO1, TPOX, D2S1338, and D19S433. MB49 murine bladder cancer plus, A549 and H520 human lung cancer cells were obtained via the UCCC tissue culture core.

\section{Animals}

Female athymic nude (nu/nu) mice (6-8 weeks) were obtained from the Animal Production Area of the National Cancer Institute (Frederick, MD) in an approved protocol.

\section{In vitro studies}

To study the binding specificity of CNR, we tested a pair of well-published model of EGFR expression, using EGFR-positive (A549) and EGFR-negative (H520) human lung cancer cell lines. In order to distinguish EGFR-positive and EGFR-negative cells by fluorescent microscope, H520-NLR cells were developed by transducing NucLight Red lentivirus (ESSEN BioScience). The stable cell line expresses nuclear restricted red fluorescent protein (excitation $588 \mathrm{~nm}$ and emission $633 \mathrm{~nm}$ ). CNR were visualized using DyLight labelled secondary antibody (488 excitation peak $493 \mathrm{~nm}$ and emission peak $518 \mathrm{~nm})$ against the C-225 (Erbitux $(2 \mathrm{mg} / \mathrm{mL})$ manufactured by ImClone LLC and Distributed by Bristol-Myers Squibb), to detect the CNR.

A mixture of A549/H520-NLR cells $\left(1 \times 10^{4}\right.$ cells/well) were seeded in 8-well chamber slides. The, cells incubated with CNR $\left(8 \times 10^{10}\right.$ particles per $\mathrm{mL}$ ) for 2 hours. Post CNR treatment, the cells were washed twice with $400 \mu \mathrm{L}$ DPBS to remove unbound CNR then probed with Dylight labelled donkey antihuman antibody for 1 hour and washed three times with DPBS. The cells with red nuclei (H520-NLR) 
A

Mixed cells in bright field

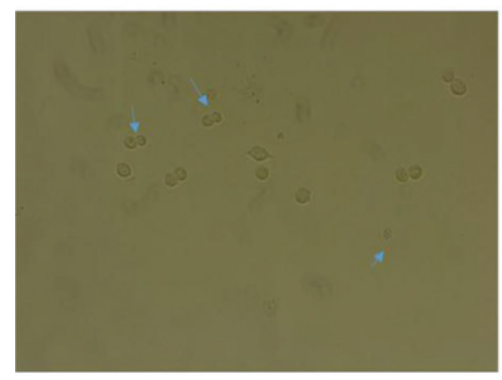

B

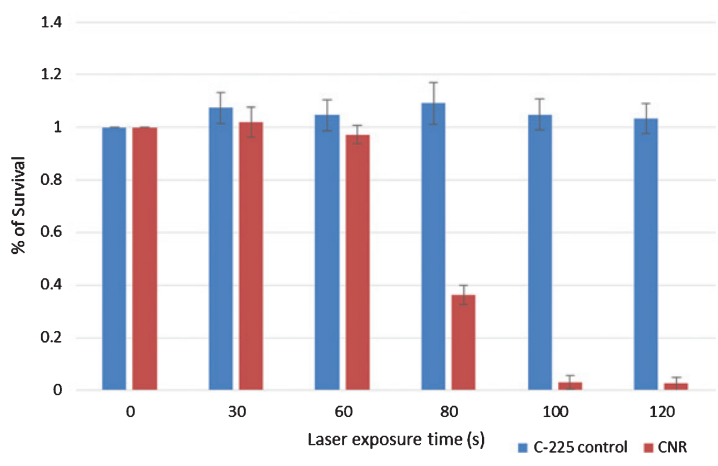

CRN specific bind to $A 549$

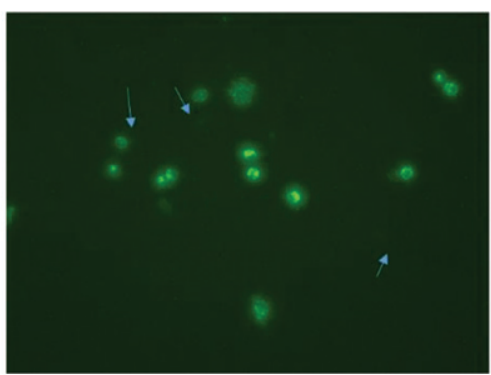

C

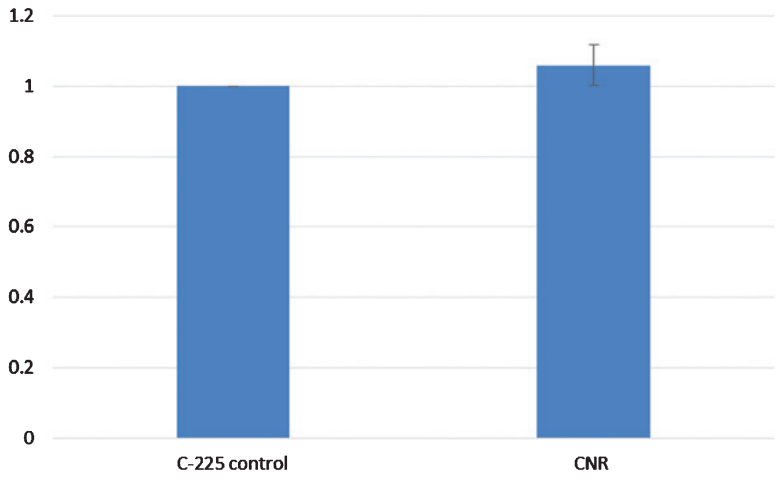

Fig. 1. In vitro testing. EGFR-negative H520 (NucLight red) were mixed with EGFR-positive A549 cells (Fig. 1A). The left panel shows bright field images of all cells, the middle panel shows green fluorescence of the CNR binding the EGFR-positive A549 cells (Donkey anti human Dylight labelled secondary antibody) and the Right panel shows the Red fluorescing H520 cells (location indicated by the blue arrows in each panel). MB49 bladder cancer cells were treated with CNR and laser at increasing exposure duration with cell survival assessed by MTT assay. Standard deviation is shown (Fig. 1B). MB49 cells were incubated with the guiding C-225 antibody and the CNR construct to assess any inherit toxicity from the CNR in the absence of laser. An MTT assay was performed after 5 days (Fig. 1C).

were visualized separately from the CNR-bound A549 dells with green fluorescent (Fig. 1A).

MB49 bladder cancer cells were trypsinized, neutralized and washed twice in DPBS. $100 \mu \mathrm{L}$ of cells $\left(6 \times 10^{7} / \mathrm{ml}\right)$ were incubated with $12 \mu \mathrm{L} \mathrm{CNR}$ $\left(8 \times 10^{11}\right.$ particles $\left./ \mathrm{ml}\right)$ for 2 hours, washed once with $1 \mathrm{ml}$ medium (OptiMEM with $3.75 \%$ FCS), centrifuged to remove supernatant and washed again with DPBS. The cells were then suspended in $200 \mu \mathrm{L}$ DPBS. Using U-bottom 96 well plate, $20 \mu \mathrm{L}$ of cells were place in each well with laser treatment at different time exposure. Immediately post laser treatment, cells were diluted in medium and MTT assay (Promega Corporation, Madison,WI, USA CellTiter AQueousOne Solution Cell Proliferation Assay) was used for cell viability assessment (Fig. 1B). To test for inherent toxicity, MB49 cells incubated with comparable levels of C-225 (1 ng/mL) and CNR for two hours, with MTT performed 5 days post treatment (Fig. 1C).

\section{Orthotopic implantation}

The orthotopic implantation procedure is similar to the one used in our previous study [12]. Briefly, the bladder was washed with phosphate buffered saline (PBS) and scratched with the catheter tip before instilling $100 \mu \mathrm{L}$ of $1.5 \times 10^{6} \mathrm{~T} 24$-Luc cells via a small catheter. The urethra was temporarily closed with a single, sterile suture at the distal portion of the urethra thus retaining the cells in the bladder for 3 hours. Xenograft implantation was confirmed by the presence of bioluminescence activity 7 days after cell implantation before subsequent nanorod and/or NIR light treatments.

\section{Bioluminescence measurement}

For bioluminescent assessment each mouse received $150 \mathrm{mg}$ luciferin/kg body weight via an intraperitoneal injection. Imaging of the mice was 
then conducted in anesthetized conditions with the IVIS $^{\circledR}$ Imaging System (MA, USA). The bioluminescent activity was quantified by software provided by Xenogen Corp. (Alameda, CA). Due to the very small size of the tumors, bioluminescent response is the primary endpoint. We have used this endpoint in previous work with an orthotopic model of bladder cancer [12] and it is a more sensitive endpoint than pathologic assessment due to the very small size of the xenograft tumors.

\section{In vivo treatment with nanorods and NIR light}

One week after cell instillation, bioluminescence activity was measured and used to distribute the mice into balanced treatment and control groups. PBS, Naked gold NanoRods (NNR), C225 (Anti-EGFR antibody), and CNR were instilled into the bladders of corresponding mouse experimental groups, following a similar procedure as described above for cancer cell instillation. Nanorods were diluted $(1: 5$ to $1: 10)$ from original production concentration to maintain a uniform concentration as determined by the optical density. This intravesical instillation was maintained in the bladder for two hours for each treatment via a temporary retention suture in the distal urethra. Next, the bladder was gently washed twice with cold PBS to remove non-specifically bound nanorods. The laser was carefully directed to the skin surface of the murine pelvis with the guidance of a visible in-fiber pilot light. The mice were treated once per week for four weeks in this manner to test the treatment efficacy and tolerance with weekly monitoring by the IVIS $^{\circledR}$ Imaging System for six weeks. To determine the laser dosing regimen, control mice without tumor cell instillation were treated with laser power densities ranging from 2.1 to $5.3 \mathrm{~W} / \mathrm{cm}^{2}$, over exposure times of 15 to 30 seconds at a fixed distance from the tip of laser fiber to the skin surface $(5.0 \mathrm{~cm})$, and under close observation.

\section{Histopathological procedures and analysis}

After 1-week of treatment, mice were sacrificed and tumors collected. Histological preparations were performed by the Prostate Diagnostic Laboratory, University of Colorado Anschutz Medical Campus. Freshly dissected tissues were bisected, fixed in $10 \%$ buffered formalin and embedded in paraffin. Five micron thick paraffin sections were deparaffinized, antigens unmasked and immunohistochemically stained for Ki-67 (Neomarkers/Thermo
Scientific, Waltham, MA; rabbit monoclonal SP6; cat\# RM-9106-SO; dilution 1:300 in TBST + 1\% BSA w/v) and cleaved Caspase 3 (Cell Signaling, Danvers, MA; rabbit polyclonal; cat\#: 9661, $1: 1000$ in TBST $+1 \%$ BSA w/v). Antigens were revealed in pH 9.5 BORG solution (Biocare Medical, Concord, $\mathrm{CA})$ for 5 minutes at $125^{\circ} \mathrm{C}(22 \mathrm{psi}$; Decloaking chamber, Biocare) with a 10 minute ambient cool down. Immunodetection was performed on the autostainer (Benchmark XT; Ventana Medical Systems, Tucson, AZ) at an operating temperature of $37^{\circ} \mathrm{C}$. Both primary antibodies were incubated for 32 minutes and detected with a modified I-VIEW DAB (Ventana) detection kit. The I-VIEW secondary antibody was replaced with a species specific polymer (Rabbit ImmPress; Full Strength; Vector Labs, Burlingame, CA; 8 minutes) and streptavidinhorseradish peroxidase was replaced with diluted Rabbit ImmPress (1:2 dilution in PBS pH 7.6; 8 minutes). All sections were counterstained in Harris hematoxylin for 2 minutes, blued in $1 \%$ ammonium hydroxide (v/v), dehydrated in graded alcohols, cleared in xylene and coverglass mounted using synthetic resin. Stains were performed by a certified histotechnologist (author EES) and the slides were reviewed by a certified pathologist (author FGLR). The pathologic evaluation of urinary bladder specimens was performed blindly to confirm the presence or absence of tumor and then compared with their corresponding bioluminescent measurements.

\section{Statistics}

The results are shown as the mean \pm standard error of the mean. The data were analyzed by single factor analysis of variance followed by the post hoc Tukey tests. Two-sided independent and paired $t$-tests were also performed where indicated. A significance level of $P<0.05$ was chosen. SPSS 22.0 (IBM) software was used for all statistical tests.

\section{RESULTS}

\section{In vitro studies}

Using well established models of EGFR expression in lung cancer, Fig. 1 demonstrates the specificity of the CNR binding to only EGFR-positive cells. The EGFR-negative H520 cells do not show any CNR binding, while the EGFR-positive A549 cells demonstrate a high level of CNR binding (Fig. 1). These data demonstrate the generalizability of this approach 
A

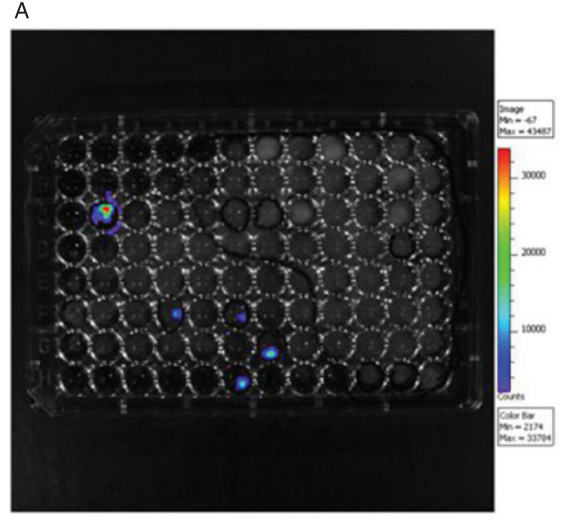

B

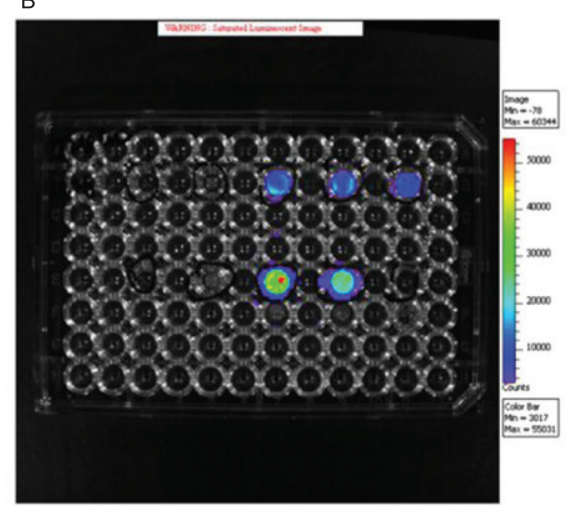

C

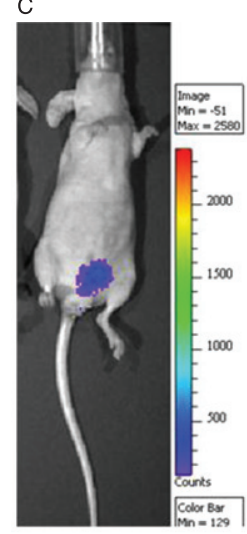

D

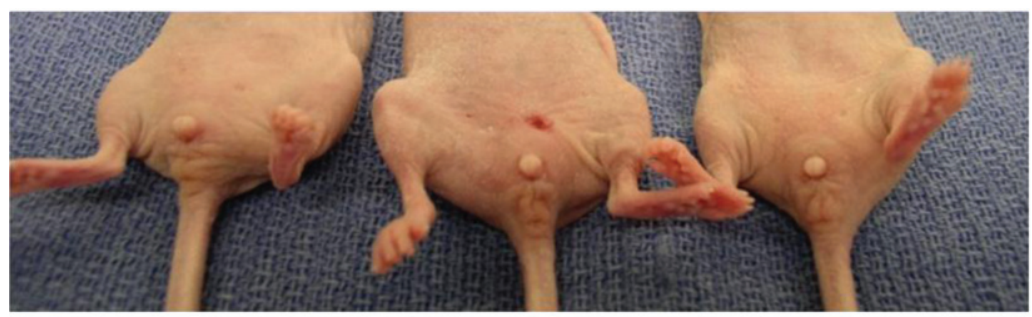

$3.2 \mathrm{~W} / \mathrm{cm}^{2} 15^{\prime \prime}$

$3.2 \mathrm{~W} / \mathrm{cm}^{2} 30^{\prime \prime}$

$2.1 \mathrm{~W} / \mathrm{cm}^{2} 30^{\prime \prime}$

Fig. 2. Establishment of new orthotopic xenograft bladder cancer model. After transfection with Lenti-luc virus, the T24 cells were seeded into 96 well plate and single clones with positive Luciferase activity were selected (Fig. 2A). These cells were cultured and Luciferase activity was further confirmed (Fig. 2B). The cells were implanted intravesically into the mouse bladders and a $90 \%$ tumor implantation rate was observed. Mice with positive tumor growth at one week were followed for two months, demonstrating tumor implantation and persistence (Fig. 2C). Representative pictures of mice at 2 days post laser treatment with the laser doses of $3.2 \mathrm{~W} / \mathrm{cm}^{2}$ for $15 \mathrm{~seconds}$ (left), $3.2 \mathrm{~W} / \mathrm{cm} 2$ for 30 seconds (middle) and $2.1 \mathrm{~W} / \mathrm{cm}^{2}$ for 30 seconds (right) (Fig. 2D). The mouse in the middle shows slight erythema and skin contraction in contrast to the other mice which show no evidence of skin changes.

to any EGFR-expressing model of cancer, as long as the surrounding normal tissue is EGFR-negative. We have previously studied the therapeutic effects of CNR + NIR laser in HTB9 bladder human cancer cells [11]. To expand on that work, the MB49 was used to study the efficacy of CNR treatment. MB49 cells represent a murine bladder cancer cell model, noting that the $\mathrm{C}-225$ antibody is reactive in murine models and MB49 cell demonstrate EGFR expression. In Fig. 1B, we see that with low levels of NIR laser application, there is no cellular killing, due to lack of thermal heating. With longer laser treatment duration, the cell death increases dramatically, but only in the cells treated in conjunction with CNR and not in those without CNR. There is no clear toxicity to CNR compared to the C-225 antibody alone.

\section{Establishment of the orthotopic xenograft urinary bladder cancer model}

T24 cells are tumorigenic [14] and have relatively high expression of EGFR [12]. Thus, we transfected
T24 cells with luciferase and selected single clones for expansion and bladder implantation, Fig. 2A and B. Bioluminescent imaging provided evidence for in vivo implantation and growth of bladder cancer cells in the nu/nu mice when assessed at day 7. Using the intravesical instillation technique described earlier, a tumor implantation rate of $90 \%$ was obtained in this model. Figure $2 \mathrm{C}$ shows positive bioluminescent signal after two months of cell implantation indicating the persistence of viable tumor in this model, and with no evidence of metastases.

\section{Optimization of safe laser dose}

Although it has been shown that photothermal ablation for urothelial carcinoma is effective in vitro, translating this approach into the in vivo setting has the risk of "bystander" toxicity from non-specific heating of neighboring urothelial tissue and also loss of efficacy from a "heat sink" effect of the surrounding tissues. Therefore, it was critical to determine a safe and effective laser dose in vivo. 
Table 1

Optimization of Laser Dose Treatment

\begin{tabular}{lllllllllll}
\hline Power Density & 1.0 & 1.0 & 1.3 & 1.3 & 2.1 & 2.1 & 3.2 & 3.2 & 4.2 & 4.2
\end{tabular} $\left(\mathrm{W} / \mathrm{cm}^{2}\right)$

$\begin{array}{lllllllllll}\text { Time (s) } & 30 & 45 & 15 & 30 & 30 & 45 & 15 & 30 & 15 & 30\end{array}$

$\begin{array}{lllllllllll}\text { Erythema Score } & 0 & 1 & 0 & 3 & 0 & 1 & 0 & 2 & 3 & 3\end{array}$

Skin toxicity was scored from 0 (no change) to 3 (erythema with chronic skin change) according to the degree of erythema.

The laser power density and the duration of laser treatment determine the integrated energy dose delivered by the laser. In the first pilot trial of the dose setting experiments, we applied the same dose level $\left(6.3 \mathrm{~W} / \mathrm{cm}^{2}\right)$ used in our in vitro experiments for a duration of 1 minute. We found that this in vitro dose was poorly tolerated and tested with lower doses of laser energy. As shown in Fig. 2D, erythema and slight but permanent tissue contraction change was observed at the power density of $3.2 \mathrm{~W} / \mathrm{cm}^{2}$ for 30 seconds, while no such changes were seen with $3.2 \mathrm{~W} / \mathrm{cm}^{2}$ for 15 seconds or at the power density of $2.1 \mathrm{~W} / \mathrm{cm}^{2}$ for 30 seconds. Table 1 summarizes the optimization of NIR laser dose according to the appearance of erythema and skin change. Based on these findings, we determined the optimal dosing and subsequent treatments were made with a power density of $2.1 \mathrm{~W} / \mathrm{cm}^{2}$ over 30 seconds.

\section{Effect of photothermal treatment on cell expression of proliferative and apoptotic biomarkers}

To minimize "heat sink" effect of urine, the bladder was drained of all urine before each treatment. We assessed the Ki67 and Cleaved-Caspase 3 expression by immunohistochemistry of the bladders after nanoparticle and laser treatment. Ki67 is known to be a proliferative biomarker associated with thermal tissue damage $[15,16]$ and has also been used to detect the effect of nanoparticle treatment [17]. Cleaved caspase- 3 has been used to discriminate between necrotic or apoptotic cell killing status after nanoparticle treatment [18] and was also assessed. As shown in Fig. 3, in the presence of CNR with the recommended dose laser treatment $\left(2.1 \mathrm{~W} / \mathrm{cm}^{2}\right.$ for 30 seconds), a mild increase of expression of Ki67 and cleaved caspase 3 was noted in the urothelium of bladder as compared with two negative controls (laser without CNR and CNR without laser), consistent with a photothermal effect. In contrast, evaluation of the tissue from the initial experiments to optimize a safe laser dose, which produced some skin toxi- city $\left(3.2 \mathrm{~W} / \mathrm{cm}^{2}\right.$ power for 30 seconds, Fig. 3A-IV) showed high levels of Ki67 expression in the underlying lamina propria of the urothelium, consistent with heat damage. Cleaved-caspase-3 staining (Fig. 3B) showed no significant changes even after high dose laser treatment (Fig. 3B-VIII). These results are consistent with previously reported values of Ki67 expression in cultured cells treated with nanorodassociated thermal ablation $[16,17]$.

\section{Photothermal effect of gold nanorod conjugates on xenograft growth}

Mice were selected for photothermal treatment after confirmation of tumor implantation, based on the presence of bioluminescent activity 7 days after the cell instillation procedure. The experiment designed here utilized three intravesical control conditions: (1) laser alone, (2) laser with NNR and (3), laser with CNR. As shown in Fig. 4A, laser plus CNR resulted in a significant reduction of luciferase activity, consistent with a therapeutic response. In contrast, the group of mice treated with laser alone had increasing bioluminescent tumor activity over time. Furthermore, CNR incubation without laser also resulted in tumor growth and persistence, but this condition was only tested in 2 mice in a demonstration fashion (Fig. 4B). Quantification of the bioluminescence levels over 6 weeks is shown in Fig. 4C. Significant changes were observed after 6 weeks of treatments (ANOVA $\mathrm{F}(2,32)=3.43$ $p=0.045)$. Post hoc tests showed that the laser plus CNR treatment (CNR+NIR light, $n=16)$ yielded lower bioluminescent activity compared to laser treatment alone $(n=14)$, Mean difference $=1045.8 \pm 400$, $p=0.035$. Furthermore, when comparing bioluminescence levels between pre-treatment and the six-week time points, mice treated with laser only showed significant increase in signal (mean difference $=520.6 \pm 233.15, p=0.044$ ). Conversely, mice treated with CNR+NIR light showed significantly lower bioluminescent activity compared to their corresponding pre-treatment levels (mean difference $=-288.50 \pm 131.87, p=0.045)$. The naked nanorods and laser treatment group (NNR+NIR light, $n=5$ ) did not show statistical significant differences. Interestingly, no significant differences were also observed between the 6-week and pretreatment time points for the NNR+NIR light group $(p=0.4)$, suggesting partial anti-tumor effect, but less activity than observed with the CNR + NIR light treatments. 


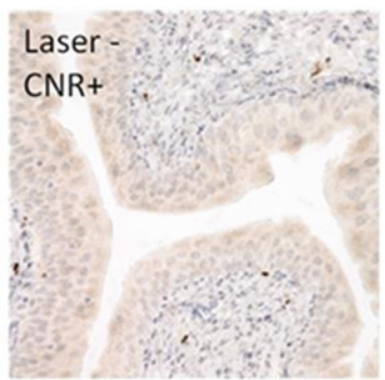

I

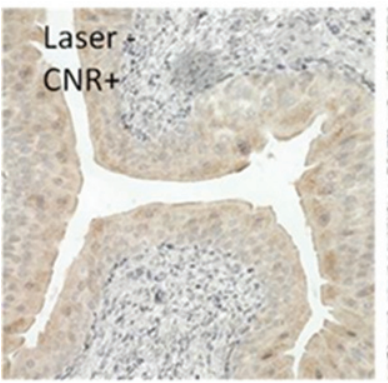

V

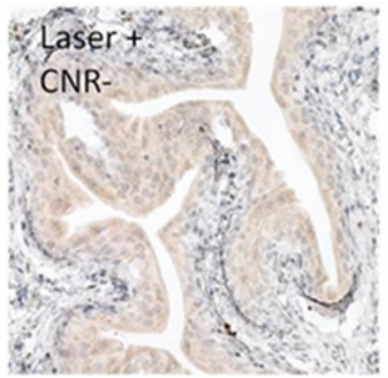

II

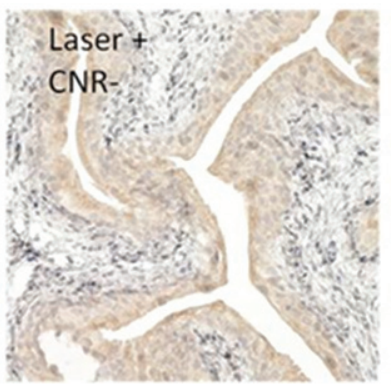

VI

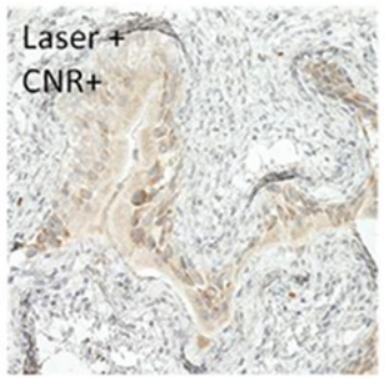

III

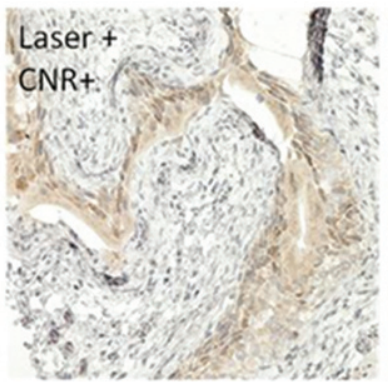

VII

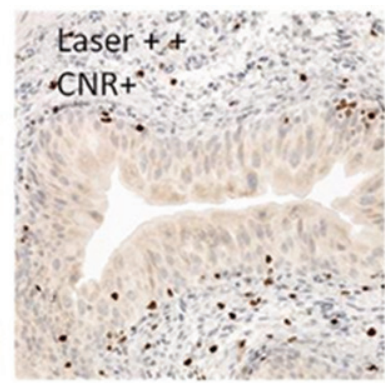

IV

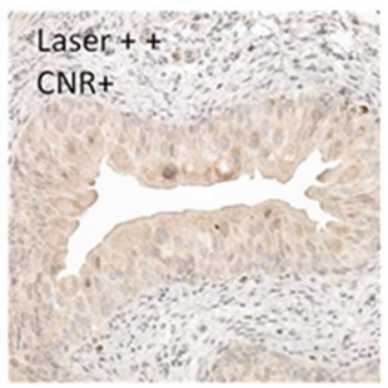

VIII

Fig. 3. Proliferative and apoptosis cell signaling after CNR and photothermal treatment. One week after photothermal treatment, the mice were euthanized and bladders were excised and subjected to formalin fixing and paraffin-embedding. Immunohistochemical stains were performed as described in the Materials and Methods section. Ki67 staining (Fig. 3A) shows an increase in nuclear expression in the bladder urothelium after treatment with the recommended laser treatment dose (power density of $2.1 \mathrm{~W} / \mathrm{cm}^{2}$ for 30 seconds) (Fig. 3A-III), while negative staining is observed in the bladder urothelium of mice from CNR only (Fig. 3A-I), and laser only (Fig. 3A-II) control treatments. Photothermal treatment at higher doses was associated with external skin toxicity (power density of $3.2 \mathrm{~W} / \mathrm{cm}^{2}$ for 30 seconds) and showed an increase in the expression of Ki67, extending into the muscularis propria (Fig. 3A-IV), consistent with a thermal treatment effect and skin changes at higher doses. Cleaved caspase-3 staining shows a similar pattern (Fig. 3B, V, VI, VII) except that there was not a positive stain in the muscularis propria observed in the mouse with higher dose of photothermal treatment (Fig. 3B-VIII).

\section{DISCUSSION}

Thermal ablation mediated by gold nanorods has been proved to effectively kill cancer cells. More recent studies have shown that gold nanorods can be modified with specific tumor-targeting antibodies to enhance their specificity and efficacy for cancer therapy [19]. In the current investigations, we have used EGFR-conjugated gold nanorods (CNR) and NIR laser therapy to specifically target orthotopic bladder xenografts in mice.

The primary outcome of our studies was the assessment of tumor bioluminescent activity over a 6-week treatment period, where CNR and laser combinatorial treatments were compared to laser treatments alone. Our studies are novel in that the delivery of the CNR was done by intravesicular instillation, allowing for the assessment of "bystander toxicity" effect in neighboring tissues and also for the "heat sink" effect of the in vivo environment, which would decrease efficacy. There are two main findings from our studies: 1) CNR plus NIR light showed a significant reduction in tumor compared to the NIR laser alone, and 2) This treatment is well tolerated in vivo, without substantial injury to the normal urothelium at the recommended dose. In fact, CNR treatment alone did not result in any decrease in tumor growth, underscoring the fact that there is no evidence that CNR treatment by itself is toxic to the cells. CNR treatment alone also did not show substantial tumor effect using in vitro models of bladder cancer in previous work [11]. Laser treatment alone also did not block tumor growth over 6 weeks, which is consistent with the previously reported lack of cellular death after NIR laser treatments on cancer cells [20].

An important concern with all classes of nanoparticles is their toxicity, which is determined by the dose, location and duration of confinement to the body. We have found that a single incubation of bladder xenografts with CNR alone did not increased the 
A
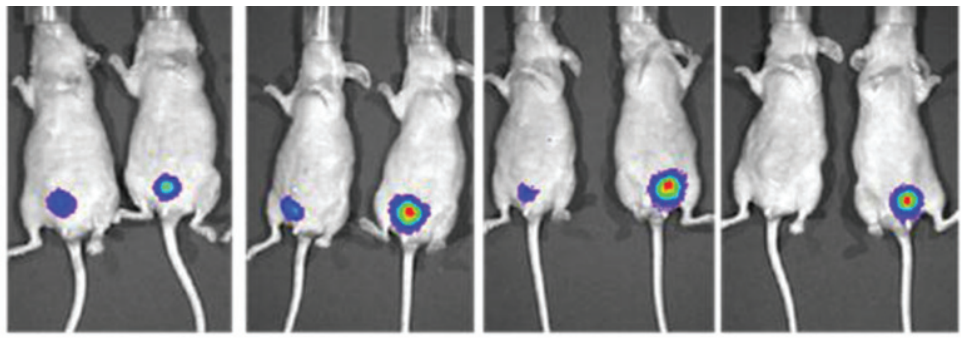

CNR

Laser
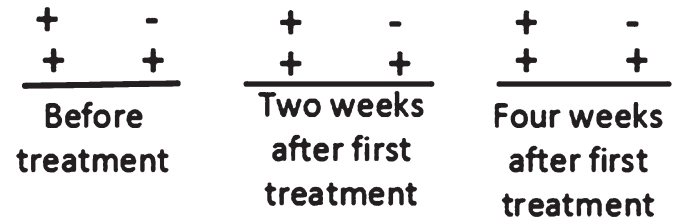

Four weeks after first

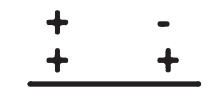
treatment treatment treatment

B
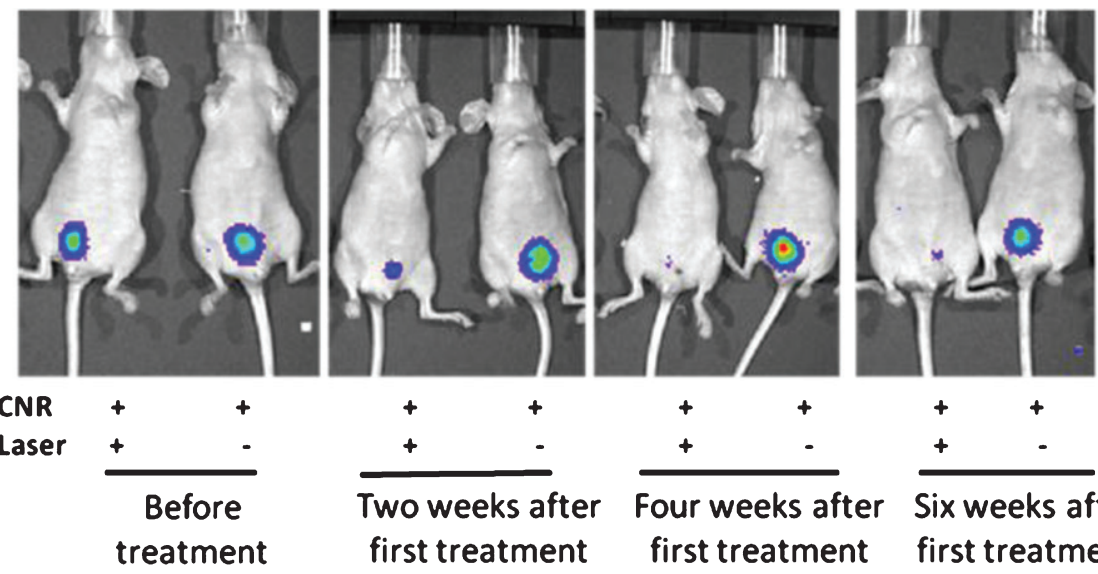

Six weeks after first treatment treatment

C

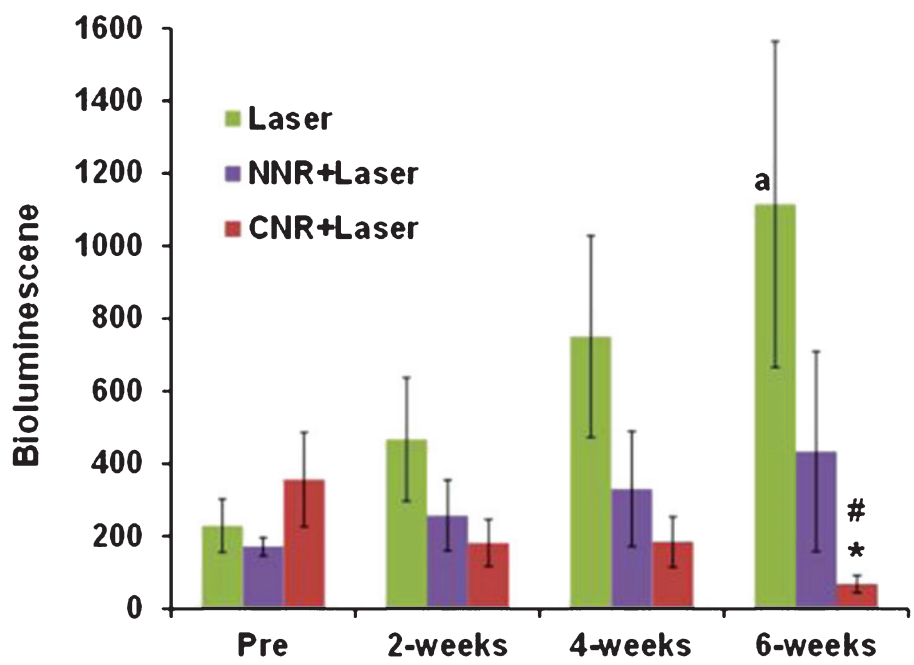

Fig. 4. In vivo Photothermal effects of nanorods. Representative mice with positive luciferase images were intravesically treated with CNR in conjunction with external delivery of NIR light once per week for four weeks, using the optimized safe dose of laser treatment (power density of $2.1 \mathrm{~W} / \mathrm{cm}^{2}$ for 30 seconds). The presence of viable tumor cells was tracked by bioluminescent imaging weekly for at least six weeks. Representative images show significant anti-tumor effect of CNR followed by NIR laser as compared with the negative controls with and without CNR followed by laser treatment (Fig. 4A) and CNR treatment with and without laser (Fig. 4B). Figure 4C shows the effects of treatments on tumor growth by luminescence units. ${ }^{*} p=0.035$ effect of CNR+Laser treatment compared to laser treatment alone at 6 weeks. ${ }^{\#} p \leq 0.04$ and ${ }^{\mathrm{a}} p=0.046$ compared to pre-treatment. $\mathrm{NNR}=$ naked nanorods, $\mathrm{CNR}=$ conjugated nanorods. 
expression of cleaved-caspase 3 , an apoptosis marker, suggesting that the CNR by themselves were not toxic to the tumor cells or the surrounding bladder tissue, consistent with what others have shown, [18]. The addition of the laser treatment did increase the stains for cleaved-caspase 3, as this treatment likely initiates apoptotic events leading to decreased xenograft tumor growth. The positive expression of the proliferation marker Ki67 in the samples treated with CNR and higher laser power was expected, since it likely reflects a repair feedback response to the laser-induced heat accumulation in the tumor and surrounding muscle $[16,17]$. However, despite these suggestive results, the mechanisms involved in the process of cell death mediated by CNR and laser treatments remain unresolved. Taken together, our findings show that this treatment is well tolerated and specific to the EGFR-expression tumor in this model.

It is important to note that the number of mice in our NNR + Laser treatment group was smaller $(n=5)$, compared to the main comparison cohorts (laser alone versus $\mathrm{CNR}+$ laser), thus hindering potential significant findings when comparing to laser treatments alone. It is possible that the NNR attached to the surface of the bladder tumor cells, without being internalized, resulted in photothermal damage after the laser treatments. In fact, other studies have shown that cancer cell death may occur in response to extracellular hyperthermia induced by gold nanorods [21]. Without the guidance of an EGFR-directed moiety, the NNR may also be less effective due to a less robust association with the EGFR-expressing tumor. Our positive results with the EGFR-conjugated nanorods plus laser treatments are proof of concept that targeted delivery of CNR to the bladder tumor cells results in well-tolerated and effective therapy. In future studies, the relationship between EGFR expression and conjugate efficacy will need to be evaluated in a broader panel of human bladder cancer cell lines and xenografts to confirm the generalizability of these results and their association with EGFR expression. The luciferase imaging results will also need to be verified independently by other methods in addition to the luciferase-based assessments performed here.

In conclusion, we describe here for the first time the use of tumor-directed nanoparticles that generate thermal energy in response to NIR light exposure in conjunction with NIR light energy as a treatment for localized bladder cancer, using an orthotopic xenograft murine urinary bladder model. The results of this investigation reveal that photothermal treatment with EGFR-directed gold nanorods has important anti-tumor effects in the in vivo setting, without gross toxicity at the recommended treatment dose. These results suggest the possibility of a similar approach in the treatment of human bladder tumors, taking advantage of current cystoscopic means to deliver therapeutic NIR laser to nanorods can also be directly administered into the bladder via routine catheterization.

\section{ACKNOWLEDGMENTS}

This work was supported by the National Cancer Institute (1R21CA152375-01) and K01 support (K01CA168934). The Pathology Core Laboratory from the Department of Pathology, University of Colorado Cancer center was utilized in this effort. This work was presented as a poster at AACR 2014 annual meeting at San Diego (April 07, 2014) number \#2728. Disclosures: Elizabeth Erin Smith and Francisco G. La Rosa have provided technical consulting to 3DBiopsy, LLC for pathology devices, Thomas Flaig and Won Park are founders of Aurora Oncology (no current commercial products). Thomas Flaig: Consulting: GTX, BN ImmunoTherapeutics; Scientific/trial support: GTX, Novartis, Bavarian Nordic, Cougar Biotechnology, Dendreon, Janssen Oncology, Medivation, Sanofi, GlaxoSmithKline, Pfizer, Bristol-Myers Squibb, Amgen, Roche/Genentech, Exelixis, Aragon, Tokai, AstraZeneca, Lilly, and Astellas. Wounjhang Park, Thomas Flaig, Xiaoping Yang, and Lih- Jen Su have a patent pending on a Multifunctional Nanomaterials for the Treatment of Cancer which is not associated with a commercial product or licensed currently.

\section{REFERENCES}

[1] Siegel RL, Miller KD, Jemal A. Cancer statistics, 2016. CA Cancer J Clin 2016;66:7-30.

[2] Kamat AM, Flaig TW, Grossman HB, et al. Expert consensus document: Consensus statement on best practice management regarding the use of intravesical immunotherapy with BCG for bladder cancer. Nat Rev Urol 2015;12:225-35.

[3] Huff TB, Tong L, Zhao Y, Hansen MN, Cheng JX, Wei A. Hyperthermic effects of gold nanorods on tumor cells. Nanomedicine (Lond) 2007;2:125-32.

[4] Kuo WS, Chang CN, Chang YT, et al. Gold nanorods in photodynamic therapy, as hyperthermia agents, and in near-infrared optical imaging. Angew Chem Int Ed Engl 2010;49:2711-5.

[5] Liu J, Yu M, Ning X, Zhou C, Yang S, Zheng J. PEGylation and zwitterionization: Pros and cons in the renal clearance and tumor targeting of near-IR-emitting gold nanoparticles. Angew Chem Int Ed Engl 2013;52:12572-6. 
[6] Longmire M, Choyke PL, Kobayashi H. Clearance properties of nano-sized particles and molecules as imaging agents: Considerations and caveats. Nanomedicine (Lond) 2008;3:703-17.

[7] Messing EM. Clinical implications of the expression of epidermal growth factor receptors in human transitional cell carcinoma. Cancer Res 1990;50:2530-7.

[8] Rotterud R, Nesland JM, Berner A, Fossa SD. Expression of the epidermal growth factor receptor family in normal and malignant urothelium. BJU Int 2005;95:1344-50.

[9] Popov Z, Gil-Diez-De-Medina S, Ravery V, et al. Prognostic value of EGF receptor and tumor cell proliferation in bladder cancer: Therapeutic implications. Urol Oncol 2004;22: 93-101.

[10] Okhunov Z, Hruby GW, Mirabile G, et al. Prospective comparison of flexible fiberoptic and digital cystoscopes. Urology 2009;74:427-30.

[11] Cho SK, Emoto K, Su LJ, Yang X, Flaig TW, Park W. Functionalized gold nanorods for thermal ablation treatment of bladder cancer. J Biomed Nanotechnol 2014;10:1267-76.

[12] Yang X, Kessler E, Su LJ, et al. Diphtheria toxin-epidermal growth factor fusion protein DAB389EGF for the treatment of bladder cancer. Clinical Cancer Research : An Official Journal of the American Association for Cancer Research 2013;19:148-57.

[13] Henderson TA, Morries LD. Near-infrared photonic energy penetration: Can infrared phototherapy effectively reach the human brain? Neuropsychiatr Dis Treat 2015;11:2191-208.
[14] Yang XH, Ren LS, Wang GP, et al. A new method of establishing orthotopic bladder transplantable tumor in mice. Cancer Biol Med 2012;9:261-5.

[15] Scholzen T, Gerdes J. The Ki-67 protein: From the known and the unknown. J Cell Physiol 2000;182:311-22.

[16] Farhangkhoee H, Cross KM, Koljonen V, Ghazarian D, Fish JS. Evaluation of Ki-67 as a histological index of burn damage in a swine model. J Burn Care Res 2012;33:e55-62.

[17] Soderstjerna E, Johansson F, Klefbohm B, Englund Johansson U. Gold- and silver nanoparticles affect the growth characteristics of human embryonic neural precursor cells. PLoS One 2013;8:e58211.

[18] Fernandez Cabada T, de Pablo CS, Serrano AM, Guerrero Fdel P, Olmedo JJ, Gomez MR. Induction of cell death in a glioblastoma line by hyperthermic therapy based on gold nanorods. Int J Nanomedicine 2012;7:1511-23.

[19] Rejiya CS, Kumar J, Raji V, Vibin M, Abraham A. Laser immunotherapy with gold nanorods causes selective killing of tumour cells. Pharmacol Res 2012;65:261-9.

[20] Choi WI, Kim JY, Kang C, Byeon CC, Kim YH, Tae G. Tumor regression in vivo by photothermal therapy based on gold-nanorod-loaded, functional nanocarriers. ACS Nano 2011;5:1995-2003.

[21] Huang HC, Rege K, Heys JJ. Spatiotemporal temperature distribution and cancer cell death in response to extracellular hyperthermia induced by gold nanorods. ACS Nano 2010;4:2892-900. 